\title{
Surgical Resection Offers Long-Term Remission in a Solitary Lung Metastasis of Differentiated Thyroid Carcinoma - A Case Report and Literature Review
}

\author{
Idit Tessler ${ }^{1,2}$ MD, MPH, Oded Cohen ${ }^{1,2}$ MD, Judith Diment ${ }^{2,3}$ MD, Yonatan Lahav ${ }^{1,2}$ MD, Doron Halp- \\ erin $^{1,2}$ MD, MPH and Taiba Zornitzki',4 MD
}

${ }^{1}$ Department of Otolaryngology, Head and Neck Surgery, Kaplan Medical Center, Rehovot, Israel

${ }^{2}$ Hadassah School of Medicine, Hebrew University, Jerusalem, Israel

${ }^{3}$ Department of Pathology, Kaplan Medical Center, Rehovot, Israel

${ }^{4}$ Diabetes, Endocrinology and Metabolic Disease Institute, Kaplan Medical Center, Rehovot, Israel

*Corresponding author: Dr. Idit Tessler, MD,MPH, Department of Otolaryngology, Head and Neck Surgery, Kaplan Medical Center; Hadassah School of Medicine, Hebrew University, Jerusalem, POB 1 Rehovot 76100, Israel, Tel: 0097252-8916133, E-mail: idit.tessler@gmail.com

\begin{abstract}
Background: Solitary pulmonary lung nodule is a rare presentation of differentiated thyroid metastatic disease with significantly increased mortality. Current data about diagnosis and treatment are limited, based mostly on case reports.

Methods: We present a case of a 56-year-old woman diagnosed with solitary pulmonary metastasis of papillary thyroid carcinoma describing the diagnostic challenge, the chosen treatment and the long-term follow-up results.

Results: The patient initially underwent a total thyroidectomy and received radioactive treatment for locally advanced papillary thyroid carcinoma. During the follow-up scans, a solitary nodule in the left lung was identified, with positive uptake in fluorodeoxyglucose PET-CT but not in an radioactive iodine uptake scan. She underwent a video-assisted thoracoscopic wedge resection. Pathology revealed metastatic papillary thyroid carcinoma. To date, five-years later, she is in a complete remission.
\end{abstract}

Conclusion: Our case demonstrates the role of surgery, not only for diagnosis but also as a single treatment for solitary lung metastasis of thyroid carcinoma providing long term remission.

\section{Keywords}

Papillary thyroid carcinoma, Solitary pulmonary metastasis, Surgical resection, Follow-up

\section{Introduction}

Differentiated Thyroid Carcinoma (DTC) is one of the most curable cancers and usually carries a good prognosis $[1,2]$. Distant metastases of DTC are relatively rare, with a wide prevalence range of $4-23 \%$ [3]. Nevertheless, it is the most common cause of thyroid cancer-related death, and is associated with a worse prognosis and a median survival of 4.1 years [4]. The most common sites for distant metastasis are in the lungs and/ or bones $[1,4]$. The classical lung involvement is diffuse lesions involving both lungs, in micronodular or miliary patterns. Solitary lung lesion is a rare presentation and is described mostly by case reports [5-7]. Hence, data concerning the diagnosis and treatment of choice is limited.

The main treatment modality for thyroid metastasis is high-dose radioactive iodine (RAI) therapy. Other treatment options include external beam radiation and targeted tyrosine kinase inhibitors for RAl-refractory cases $[3,8]$. Metastasectomy have been reported mostly with regard to bone metastasis, more as a palliative measure rather than a curative one [2,9]. The role of pulmonary metastasectomy remains unclear, with only scant literature based on case reports and retrospective

Citation: Tessler I, Cohen O, Diment J, Lahav Y, Halperin D, et al. (2020) Surgical Resection Offers LongTerm Remission in a Solitary Lung Metastasis of Differentiated Thyroid Carcinoma - A Case Report and Literature Review. Int Arch Endocrinol Clin Res 6:020. doi.org/10.23937/2572-407X.1510020

Accepted: May 16, 2020: Published: May 18, 2020

Copyright: (c) 2020 Tessler I, et al. This is an open-access article distributed under the terms of the Creative Commons Attribution License, which permits unrestricted use, distribution, and reproduction in any medium, provided the original author and source are credited. 
data only [10]. Only a few reports on pulmonary metastasectomies have described a cure $[7,11]$. Although the follow-up was relatively short for a cure estimation from papillary thyroid carcinoma (PTC), as recurrences may appear years following the treatment.

Here we present a five-year follow-up of a 56-yearold woman diagnosed with a PTC solitary pulmonary metastasis, successfully treated with surgical resection.

\section{Case Report}

A 56-year-old Caucasian woman was diagnosed with a solitary pulmonary nodule found during a follow up for PTC. Four years prior, the patient was treated with mercaptizole for Graves' disease, and was followed up in an institutional endocrinology clinic. She underwent a right hemithyroidectomy due to the presence of a suspicious thyroid nodule classified as Bethesda category III) on fine needle aspiration. The pathology examination demonstrated a $15 \mathrm{~mm}$ PTC with macroscopic strap muscle invasion. The patient underwent a completion thyroidectomy, with no additional tumor foci. The patient was treated with $150 \mathrm{mCi}$ RAl after withdrawal of thyroid hormone treatment. Post-treatment thyroglobulin levels was $0.2 \mathrm{ng} / \mathrm{mL}$ (normal range 0.8-55.00 $\mathrm{ng} / \mathrm{mL}$ ), and her thyroglobulin Ab level was $216 \mathrm{IU} / \mathrm{ml}$ (normal range 10.00-40.00 IU/ml). Five months later, a follow-up Thyrogen stimulated radioiodine scan with SPECT/CT was performed, demonstrating an indetermi- nate finding in the right lung lobe, for which a CT scan was recommended. The patient underwent a chest $\mathrm{CT}$ scan which showed a $21 \times 16 \mathrm{~mm}$ mass in the left lung upper lobe (Figure 1), with no lesion in the right lung. The lesion in the left lung demonstrated a pathological fluorodeoxyglucose (FDG) uptake in a PET-CT scan (Figure 2). Seven month after the surgery, the thyroglobulin and thyroglobulin $A B$ were undetectable. At this point, the differential diagnosis included primary lung cancer or a metastatic lesion of thyroid origin. A video-assist-

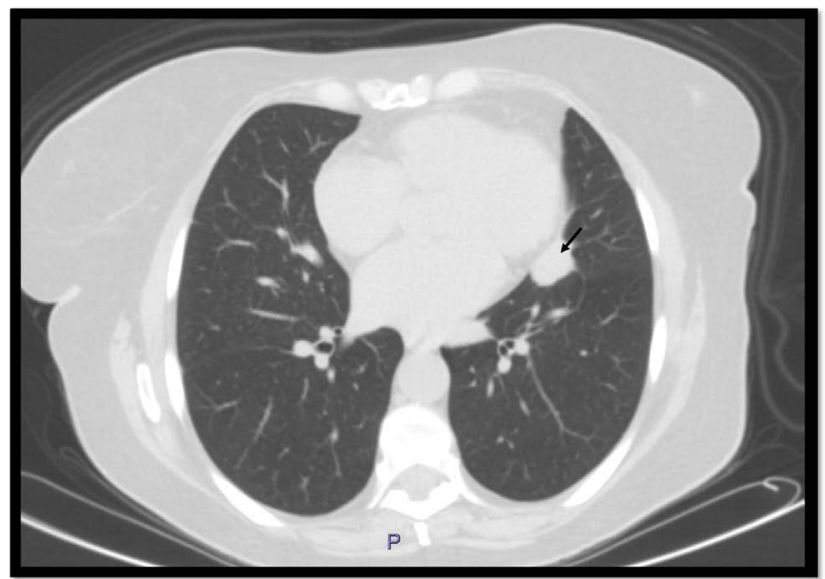

Figure 1: A Computed Tomography (CT) scan of the chest, axial section. A $21 \times 16 \mathrm{~mm}$ solid lesion in the lingula precardial area of the left upper lobe (arrow) is demonstrated, without evidence for lymph nodes enlargement.

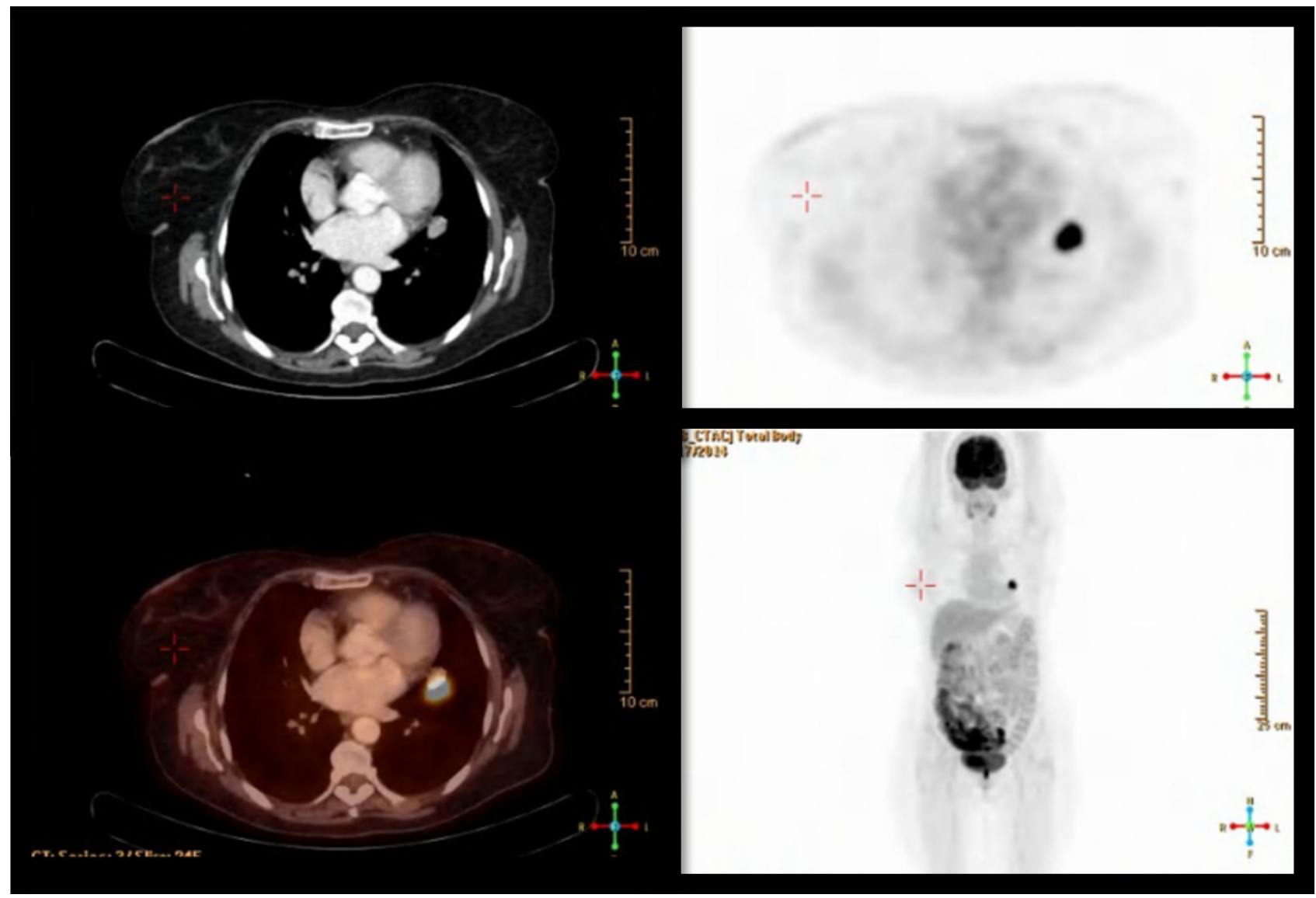

Figure 2: PET/CT scan with FDG. A 22X14 mm lesion with spiky borders is demonstrated in the left upper lobe paramediastinal, in close proximity with the pericard. No pathological uptake of FDG was demonstrated in the neck region. 


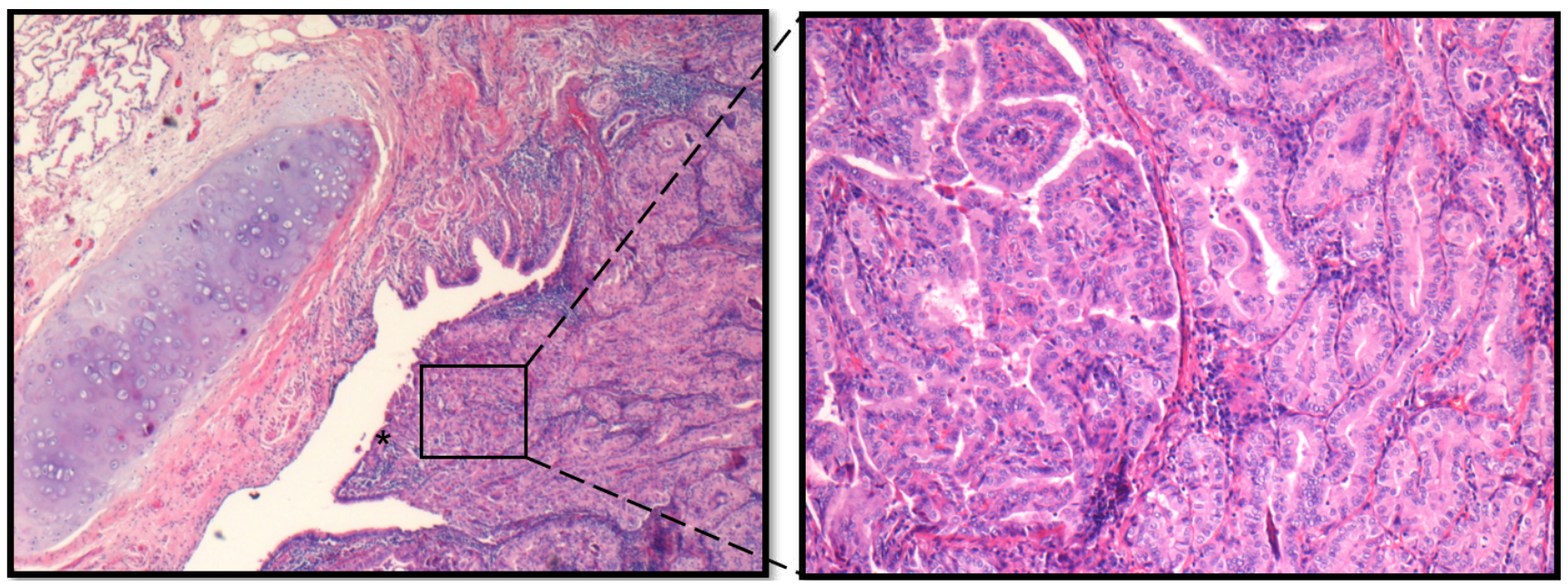

Figure 3: (A) A $25 \mathrm{~mm}$ focus of metastatic papillary thyroid carcinoma in the lung, adjacent to a bronchus and involving the bronchial mucosa (asterisk); (B) Magnification of the tumor foci demonstrating papillary structures. Hematoxylin and Eosin stain.

ed thoracoscopic excisional biopsy was performed. The pathology examination revealed a $25 \mathrm{~mm}$ focus of metastatic papillary thyroid carcinoma, resected with clear margins (Figure 3). Staining was positive for thyroid Transcription Factor-1 (TTF1), thyroglobulin and cytokeratin (CK) 7. The surgery and the postoperative course were uneventful. The postoperative FDG PET-CT scan confirmed resolution. From the ambulatory follow-up to the current date, the patient is in complete remission without any structural or biochemical evidence recurrence for a period of 61 months.

\section{Discussion}

About one-third of advanced DTC have metastatic lesions with low avidity for radioiodine treatment at the time of diagnosis or during the progression of the disease, when the ability to concentrate radioiodine is lost. In our patient, the thyroid carcinoma presented as a combination of RAI sensitive neck disease and non-avid iodine solitary lung metastasis. Solitary metastasis is relatively uncommon and was previously reported mostly in the lung, bone and brain [1]. The pre-operative diagnosis of a solitary lung metastasis might be misleading and requires a high index of suspicion. In this case, the lesion was discovered several months following the initial surgery, without evidence of previous regional spread, despite significant thyroid disease with strap muscle involvement.

DTC typically metastasizes through lymphatic spread leading to stepwise progression from primary to regional and then distant metastasis. The presentation here of solitary lung metastasis without regional disease in the neck may suggests that the metastatic spread was hematogenous, thus 'skipping' the regional neck.

RAl is currently the treatment of choice for thyroid cancer metastases. It is considered effective for microscopic metastatic disease but may not be sufficient for macroscopic metastases [12], which require extremely high radiation doses to reach a tumoricidal effect
$[11,13]$. High dose RAl for pulmonary metastasis has been associated with impaired pulmonary functions and pulmonary fibrosis [14]. Furthermore, a pulmonary macronodular lesion was shown to be a predictor for non-131-avid metastases, as seen in this case report as well. This leads to a lower response rate and reduced survival rates $[1,3]$.

When the metastatic disease is not amenable to RAI therapy, the National Comprehensive Cancer Network (NCCN) guidelines suggests local treatment, such as surgical resection or radiation [2]. Previous retrospective series on the role of metastasectomy for DTC metastatic diseases have showed beneficial effects, with regard to all sites, including the lung [9-11]. Pak, et al. reviewed 47 surgeries for distant metastasis in advanced thyroid cancer, including 12 pulmonary metastasectomies used in conjunction with RAl therapy, and found it beneficial [11]. In Porterfield et al. study, pulmonary resection for thyroid metastasis was safe with low morbidity and mortality and demonstrated improved long-term survival [10]. Here, we report on a five-year disease-free follow-up after metastasectomy as a single modality. Previous studies have suggested criteria for pulmonary metastasectomy: (1) Absence of widely disseminated disease; (2) Eradication of the primary tumor; (3) No medical contraindications for surgery; and (4) Adequate pulmonary function that can tolerate resection of all documented disease $[11,15]$. These terms were verified during the course of this case report, leading to remission by surgery.

\section{Conclusion}

In a solitary lung metastasis, surgery appears to be pivotal for the diagnosis and can achieve long-term remission when used as a single modality. Surgery allows the avoidance of RAl large doses and related complications. Thus, surgery as an additional treatment option should be considered in patients with solitary metastases of thyroid carcinoma. 


\section{Disclosure}

The authors report no conflicts of interest.

\section{References}

1. Sampson E, Brierley JD, Le LW, Rotstein L, Tsang RW (2007) Clinical management and outcome of papillary and follicular (differentiated) thyroid cancer presenting with distant metastasis at diagnosis. Cancer 110: 1451-1456.

2. Haddad RI, Nasr C, Bischoff L, Busaidy NL, Byrd D, et al. (2018) NCCN Guidelines Insights: Thyroid Carcinoma, Version 2.2018. J Natl Compr Canc Netw 16: 1429-1440.

3. Song HJ, Qiu ZL, Shen CT, Wei WJ, Luo QY (2015) Pulmonary metastases in differentiated thyroid cancer: efficacy of radioiodine therapy and prognostic factors. Eur $\mathrm{J}$ Endocrinol 173: 399-408.

4. Shoup M, Stojadinovic A, Nissan A, Ghossein RA, Freedman S, et al. (2003) Prognostic indicators of outcomes in patients with distant metastases from differentiated thyroid carcinoma. J Am Coll Surg 197: 191-197.

5. Shigematsu $\mathrm{H}$, Andou A, Teramoto A, Matsuo K, Oda W, et al. (2009) Solitary Lung Metastasis Diagnosed 30 Years After Surgery for Thyroid Cancer. Ann Thorac Surg 88: 2016-2017.

6. Fonseca $P$ (1999) Thyroid Lung Metastasis Diagnosed 47 Years After Thyroidectomy. Ann Thorac Surg 67: 856-857.

7. Strate SM, Lee EL, Childers JH (1984) Occult papillary carcinoma of the thyroid with distant metastases. Cancer 54 : 1093-1100.
8. Chung J-K, Cheon GJ (2014) Radioiodine Therapy in Differentiated Thyroid Cancer: The First Targeted Therapy in Oncology. Endocrinol Metab 29: 233-239.

9. Stojadinovic A, Shoup M, Ghossein RA, Nissan A, Brennan MF, et al. (2002) The role of operations for distantly metastatic well-differentiated thyroid carcinoma. Surgery 131: 636-643.

10. Porterfield JR, Cassivi SD, Wigle DA, Shen KR, Nichols FC, et al. (2009) Thoracic metastasectomy for thyroid malignancies. Eur J Cardiothorac Surg 36: 155-158.

11. Pak H, Gourgiotis L, Chang WI, Guthrie LC, Skarulis MC, et al. (2003) Role of metastasectomy in the management of thyroid carcinoma: the NIH experience. J Surg Oncol 82: 10-18.

12. Martin S, Maurice T, Vathaire FD, Catherine $H$, Gardet $P$, et al. (1986) Long-Term Results of Treatment of 283 Patients with Lung and Bone Metastases from Differentiated Thyroid Carcinoma. J Clin Endocrinol Metab 63: 960-967.

13. Maxon HR, Thomas SR, Hertzberg VS, Kereiakes JG, Chen IW, et al. (1983) Relation between effective radiation dose and outcome of radioiodine therapy for thyroid cancer. N Engl J Med 309: 937-941.

14. Jang EK, Kim WG, Kim HC, Huh JW, Kwon H, et al. (2015) Changes in the Pulmonary Function Test after Radioactive lodine Treatment in Patients with Pulmonary Metastases of Differentiated Thyroid Cancer. PLoS One 10: e0125114.

15. Khan JH, McElhinney DB, Rahman SB, George TI, Clark $\mathrm{OH}$, et al. (1998) Pulmonary metastases of endocrine origin: the role of surgery. Chest 114: 526-534. 